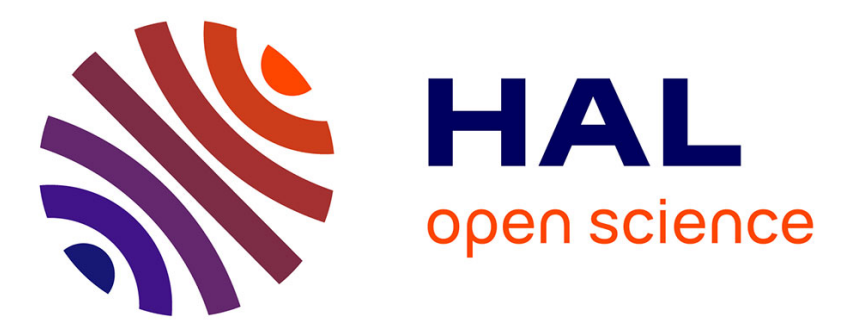

\title{
Marionette Meshes: modelling free-form architecture with planar facets
}

Romain Mesnil, Cyril Douthe, Olivier Baverel, Bruno Léger

\section{To cite this version:}

Romain Mesnil, Cyril Douthe, Olivier Baverel, Bruno Léger. Marionette Meshes: modelling free-form architecture with planar facets. International Journal of Space Structures, 2017, 32 (3-4), pp.184-198. 10.1177/0266351117738379 . hal-01616999

\section{HAL Id: hal-01616999 \\ https://hal.science/hal-01616999}

Submitted on 7 Nov 2017

HAL is a multi-disciplinary open access archive for the deposit and dissemination of scientific research documents, whether they are published or not. The documents may come from teaching and research institutions in France or abroad, or from public or private research centers.
L'archive ouverte pluridisciplinaire HAL, est destinée au dépôt et à la diffusion de documents scientifiques de niveau recherche, publiés ou non, émanant des établissements d'enseignement et de recherche français ou étrangers, des laboratoires publics ou privés. 


\section{Marionette meshes: modelling free-form architecture with planar facets}

\author{
Romain Mesnil $^{1,2}$, Cyril Douthe ${ }^{2}$, Olivier Baverel ${ }^{2}$, Bruno Leger ${ }^{1}$
}

\begin{abstract}
We introduce an intuitive method, called Marionette by the authors, for the modelling of free-form architecture with planar facets. The method takes inspiration from descriptive geometry and allows to design complex shapes with one projection and the control of elevation curves. The proposed framework achieves exact facet planarity in real-time, and considerably enriches previous geometrically-constrained methods for free-form architecture. A discussion on the design of quadrilateral meshes with a fixed horizontal projection is first proposed, and the method is then extended to various projections and patterns.

The method used is a discrete solution of a continuous problem. This relation between smooth and continuous problem is discussed and shows how to combine the marionette method with modelling tools for smooth surfaces, like NURBS or T-splines. The result is a versatile tool for shape modelling, suited to engineering problems related to free-form architecture.
\end{abstract}

\title{
Keywords
}

Descriptive geometry, Marionette meshes, fabrication-aware design, structural morphology, architecture, mesh planarisation

\section{Introduction}

The design of complex architectural shapes has benefited from great advances from the computer graphics community in the last decade. For instance, significant efforts were made to develop numerical methods for the covering of free-form surfaces with planar panels. These methods differ from the common knowledge of architects and engineers. Although properly implemented methods are efficient and relatively simple to use, they remain black boxes providing little insight on the nature of the design constraints imposed by facet planarity. The technique proposed in the present article aims thus at modelling efficiently meshes with planar facets and at providing the designers with an understanding of the design space. The proposed method takes inspiration from descriptive geometry, a tool used by architects for centuries, and turns it into a real-time design tool for PQ-meshes.

\subsection{Prior works}

Geometrically-constrained approach Planar quadrilaterals have been identified by practicians as an important optimisation target for the construction of double curved surfaces, as they avoid using curved panels (Glymph et al. 2004). Several techniques for generating exact planar quadrilateral meshes were proposed, mostly relying on affine transformations, which preserve planarity, a notion illustrated in (Pottmann et al. 2007). For example, Scale-trans surfaces, introduced in (Schober 2002) use composition of two affine transformations: translation and homothetic transformations. The designer control the shape with two curves, making the process highly intuitive. Despite formal limitations, these shapes have been used in many projects.

The term of geometrically-constrained design approach has been introduced by (Bagneris et al. 2008). Constrained geometric approaches use shapes that are well-known and can be rationalised efficiently, for example towards a high repetition of nodes or panels (Mesnil et al. 2015). More recenty, other design strategies exploring more

\footnotetext{
${ }^{1}$ Bouygues Construction, Guyancourt, France

${ }^{2}$ Laboratoire NAVIER, UMR 8205, École des Ponts, IFSTTAR, CNRS, UPE, Champs-sur-Marne, France

Corresponding author:

Romain Mesnil, École des Ponts ParisTech 8, avenue Blaise Pascal, 77420 Champs-sur-Marne, France.

Email: romain.mesnil@enpc.fr
} 
complex shapes based on Möbius transformations and inspired by (Bobenko and Huhnen-Venedey 2012) have been proposed (Mesnil et al. 2017b). The idea to use groups of transformations to study geometrical properties of surfaces is not new (Klein 1893), but recent applications to architecture show that this has a great potential. Generally speaking, these methods suffer however from a lack of flexibility and form a restricted design space. New geometricallyconstrained techniques merging fabrication and structural considerations have been proposed recently and extend the potential of classical methods. For example, an elegant design methodology based on planar pre-stressed networks through Airy stress function allowed Adrianssens et al. to design a funicular irregular gridshell with planar facets Adriaenssens et al. (2012). The lack of flexibility of usual modelling techniques led to the introduction of postrationalisation strategies in order to cover arbitrary shapes with planar quadrilaterals (Liu et al. 2006).

Optimisation-based shape exploration Most recent methods propose hence to explore design space of feasible solutions for a given mesh topology with the help of optimisation techniques (Deng et al. 2015; Yang et al. 2011). The mesh is interactively deformed by the user with the help of control handles. The overall smoothness is checked with discrete functions of the vertices. To go further, an efficient solver handling quadratic constraints was presented in (Tang et al. 2014) and used in (Jiang et al. 2014).

Projections and subspace exploration are efficiently used for constrained-based optimisation in (Bouaziz et al. 2012; Deng et al. 2013, 2015). These methods provide a great design freedom, but illustrations shown in the cited references are local,handle-based, deformations of meshes. Local deformations of shapes are of particular interest in the computer graphics community, but architectural modelling paradigms are generally thought as ways to steer a shape as a whole.

The idea here is to use the notion of projection, which is commonly used in architecture, especially with plane view and elevations, and to link subspace exploration techniques with representation techniques based on projections in architecture.

Descriptive Geometry Descriptive geometry is a technique of shape representation invented by French mathematician Gaspard Monge (Monge 1798; Javary 1881). It is based on planar orthogonal projections of a solid. The planes, in which the projections are done, are usually the horizontal and vertical planes. Figure 1 is a typical drawing of descriptive geometry: it describes an ellipsoid with a plane view, displayed with some elevations. The curve network corresponds to the horizontal projection of lines of curvature (Leroy 1857).

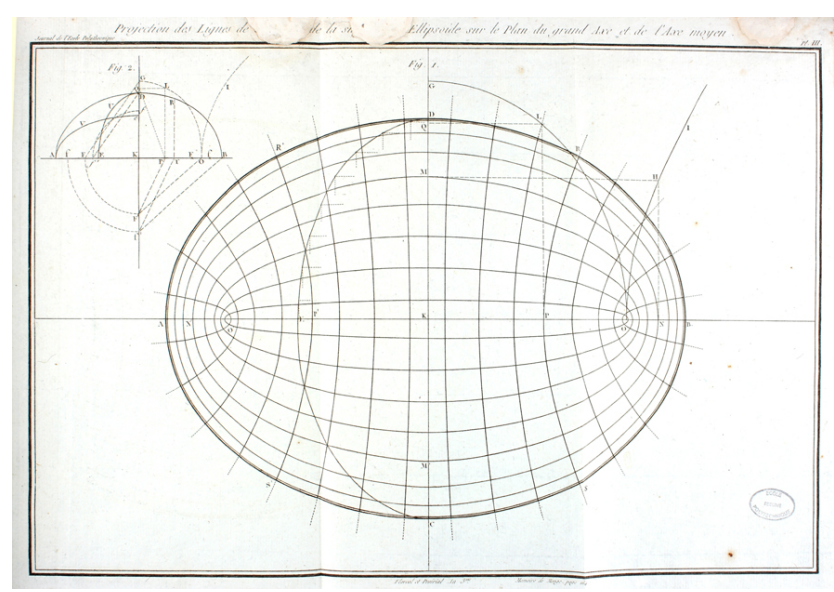

Figure 1. Lines of curvatures of an ellipsoid with descriptive geometry (Leroy 1857).

Because architectural objects have to deal mainly with gravity and vertical forces, it makes naturally sense to separate projections in vertical and horizontal planes. The idea to use these projections to guide structural design was used recently in computational frameworks based on the Thrust Network Analysis (Block and Ochsendorf 2007) where compression-only structures are found from a planar network at equilibrium (Rippmann et al. 2012; Miki et al. 2015). The objective of this paper is to show that descriptive geometry can be turned into a general tool for the design of PQ meshes and their structural optimisation. The method, called Marionette method is presented in Section 2, where the relation between smooth and discrete geometry for PQ-meshes is discussed. Section 3 explores then some applications in architecture. Section 4 shows finally the generality of the proposed method, which can be extended to meshes other than regular quadrilateral meshes and therefore constitute a promising versatile tool to intuitively integrate fabrication constraints into architectural design.

\section{Marionette Meshes}

\subsection{Marionette Quad}

The principles of descriptive geometry can be transposed to architectural shape modelling. The use of appropriate projections provides a simple interpretation of the problem of meshing with flat quadrilaterals. For simplification, we discuss the case of a projection in the $(X Y)$ plane in this section: the generalisation to other projections is illustrated in Section 4. 
Consider first Figure 2: four points have a prescribed plane view $\mathbf{A B C D}$ in the horizontal plane $\left(P_{1}\right)$. Three points $\mathbf{A}^{\prime}$, $\mathbf{B}^{\prime}$ and $\mathbf{D}^{\prime}$ have prescribed heights $z_{A}, z_{B}$ and $z_{D}$. In general, there is only one point $\mathbf{C}^{\prime}$ with the imposed projection $\mathbf{C}$ so that $\mathbf{A}^{\prime} \mathbf{B}^{\prime} \mathbf{C}^{\prime} \mathbf{D}^{\prime}$ is planar.
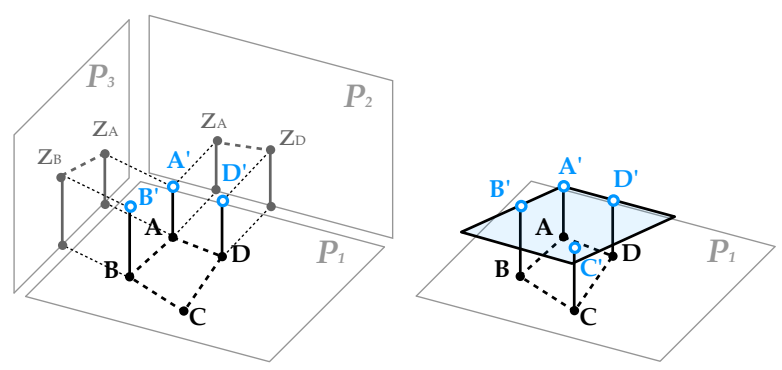

Figure 2. Creation of a Marionette Quad with a plane view and two elevations.

The planarity constraint reads:

$$
\operatorname{det}\left(\mathbf{A}^{\prime} \mathbf{B}^{\prime}, \mathbf{A}^{\prime} \mathbf{C}^{\prime}, \mathbf{A}^{\prime} \mathbf{D}^{\prime}\right)=0
$$

Expressing coordinates in a cartesian frame of $\left(P_{1}\right), \quad$ and writing $d_{B C}=\operatorname{det}_{2 \mathrm{D}}(\mathbf{A B}, \mathbf{A C}), \quad d_{B D}=$ $\operatorname{det}_{2 \mathrm{D}}(\mathbf{A B}, \mathbf{A D})$ and $d_{D C}=\operatorname{det}_{2 \mathrm{D}}(\mathbf{A D}, \mathbf{A C})$, if the points $A, B$ and $D$ are not aligned, then, one gets:

$\left(z_{C}-z_{A}\right)=\left(\frac{d_{B C}}{d_{B D}}\right) \cdot\left(z_{D}-z_{A}\right)+\left(\frac{d_{D C}}{d_{B D}}\right) \cdot\left(z_{B}-z_{A}\right)$

Figure 2 shows vertical lines used for construction, recalling the strings of a marionette, which gives the name marionette quad. Note that the system is under-constrained if the points $A, B$ and $D$ are aligned: in that case, the vectors $\mathbf{A B}$ and $\mathbf{A D}$ are colinear and $d_{B D}$ is equal to zero. This configuration corresponds to vertical a quad. A projection in the horizontal plane thus allows only for the modelling of height fields. This limitation can be overcome by using other projections (see Section 4).

\subsection{Regular Marionette Meshes}

Consider now a quadrangular mesh without singularity as depicted in Figure 3. The plane view in the horizontal plane is fixed, and two intersecting curves are defined on the projection planes $P_{2}$ and $P_{3}$. Then, provided that the planar projection admits no degenerated quad (i.e. quad where three points are colinear), equation (2) can be propagated through a strip, and by there, through the whole mesh. Indeed, on the highlighted strip of Figure 3, the first quad (top left) has three prescribed $z$-values, and equation (2) can be used and so forth. The same applies for all the quads of the strip.
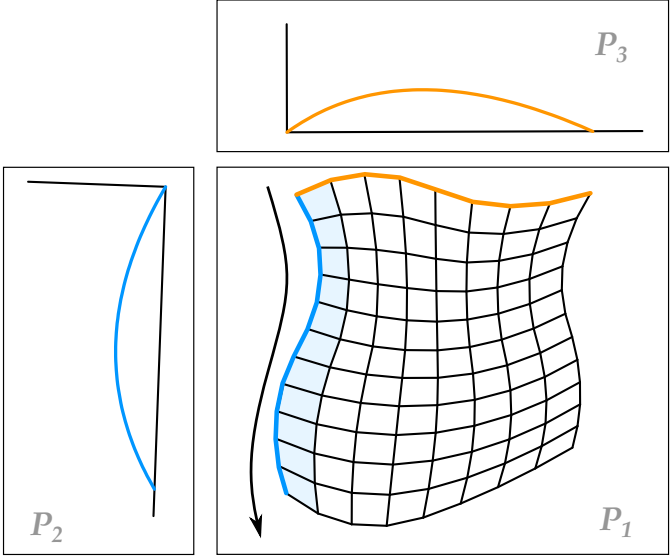

Figure 3. Two elevations and a planar projection define a unique Marionette Mesh.

For a $N \times M$ mesh, the propagation requires $N M$ applications of equation (2), the memory needed is $3 N M$. The marionette technique guarantees hence that the number of operations varies linearly with the number of nodes within a structure. The method performs thus in real-time even for meshes with thousands of nodes, as discussed in Section 3.1.

\subsection{Link with smooth geometry}

Partial differential equation The proposed method has some interesting relations with smooth geometry. The problem of covering curved shapes with planar panels is linked with the integration of conjugate curves networks (Liu et al. 2006; Bobenko and Suris 2008). Conjugate networks correspond to parameterisations $(u, v)$ satisfying the following equation (Bobenko and Suris 2008):

$$
\operatorname{det}\left(\partial_{\mathbf{u}}, \partial_{\mathbf{v}}, \partial_{\mathbf{u v}}^{\mathbf{2}}\right)=0
$$

Consider now that the components in $x$ and $y$ are fixed, like in the problem solved by the Marionette technique. We are looking for the height functions $f^{z}$ satisfying equation 3 . Adopting the notation $f_{u}$ to denote differentiation of $f$ with respect to $u$, equation (3) is reformulated into:

$$
\operatorname{det}\left(\begin{array}{ccc}
f_{u}^{x} & f_{v}^{x} & f_{u v}^{x} \\
f_{u}^{y} & f_{v}^{y} & f_{u v}^{y} \\
f_{u}^{z} & f_{v}^{z} & f_{u v}^{z}
\end{array}\right)=0
$$

Equation (4) is defined if the parameterisation in the plane $(X Y)$ is regular, which means if the study is restricted to height fields. We expand (4) using adjugate matrices:

$$
\left|\begin{array}{cc}
f_{u}^{x} & f_{v}^{x} \\
f_{u}^{y} & f_{v}^{y}
\end{array}\right| f_{u v}^{z}+\left|\begin{array}{cc}
f_{v}^{x} & f_{u v}^{x} \\
f_{v}^{y} & f_{u v}^{y}
\end{array}\right| f_{u}^{z}-\left|\begin{array}{cc}
f_{u}^{x} & f_{u v}^{x} \\
f_{u}^{y} & f_{u v}^{y}
\end{array}\right| f_{v}^{z}=0
$$


Equation (5) is a second order linear equation in $f^{z}(u, v)$. The only term of second order is $f_{u v}^{z}$ : the equation is thus hyperbolic. Hyperbolic equations often correspond to the propagation of informations in a system (think of the wave equation). It is thus no surprise that the marionette method corresponds to a propagation algorithm. Loosely speaking, it can be shown that solutions of hyperbolic equations retain discontinuities of initial conditions. The smoothness of the shape obtained by the marionette method is thus dependent on the smoothness of the input data (plane view and elevation curves) (Alinhac 2009).

Boundary conditions With the marionette method, we prescribe the values of $f^{z}$ on two boundaries. Mathematically, we choose two functions $f_{1}(v)$ and $f_{2}(u)$ which correspond to the height of the two guide curves:

$$
\left\{\begin{aligned}
f^{z}\left(u=u_{0}, v\right) & =f_{1}(v) \\
f^{z}\left(u, v=v_{0}\right) & =f_{2}(u) \\
f_{2}\left(u_{0}\right) & =f_{1}\left(v_{0}\right)
\end{aligned}\right.
$$

The last equality correponds to a compatibility condition between equation $f_{1}(v)$ and $f_{2}(u)$, so that the height of $f^{z}\left(u_{0}, v_{0}\right)$ is known without ambiguity. This equation corresponds to an integration of the second member of equation (7). Writing $f_{3}(u)=\frac{\partial f_{2}}{\partial u}(u)$, we have:

$$
\left\{\begin{aligned}
f^{z}\left(u=u_{0}, v\right) & =f_{1}(v) \\
\frac{\partial f^{z}}{\partial u}\left(u, v=v_{0}\right) & =f_{3}(u)
\end{aligned}\right.
$$

We see now that we specify the height of a guide curve and the slope on the second curve. This kind of boundary condition based on both values and derivatives is called Cauchy boundary condition and is particularly suited for hyperbolic equations (Alinhac 2009). The smooth problem solved by the marionette method is thus a classical problem in the theory of partial differential equations. Classical results on the existence, uniqueness and regularity of solution can be applied, even though it is not the purpose of this paper.

\subsection{Marionette Meshes with singularities}

The modelling of complex shapes requires the introduction of internal vertices with a valence other than four, called singularities in the following. For example, the mesh displayed in Figure 4a has one singularity: the central node has a valence of six. The mesh can be subdivided into six patches with no inner singularity (in blue and white). This kind of procedure can be applied to any quad-mesh. Each patch is a regular mesh, and the Marionette technique can be applied. There are however restrictions on the curves used as guide curves due to compatibility between patches. For example, in Figure $4 \mathrm{a}$, it is clear that the six curves attached to the singularity can be used as guides for the six patches, whereas choosing the 12 curves on the perimeter over-constrain the problem.

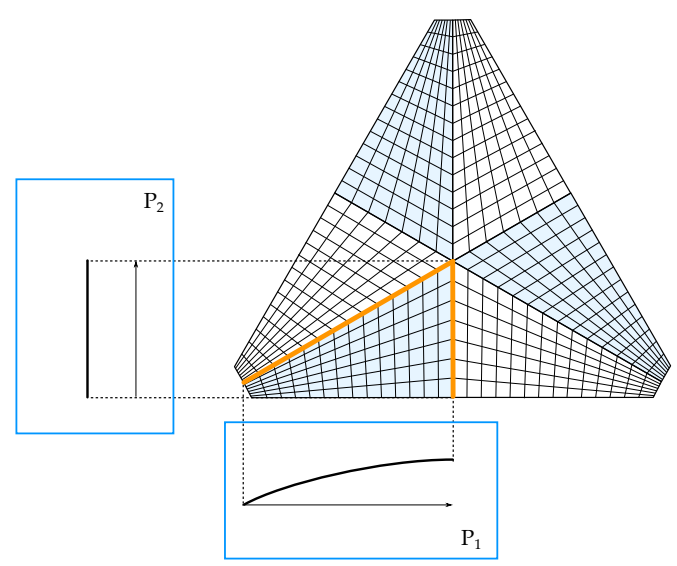

(a) Decomposition of a complex mesh into simple patches.

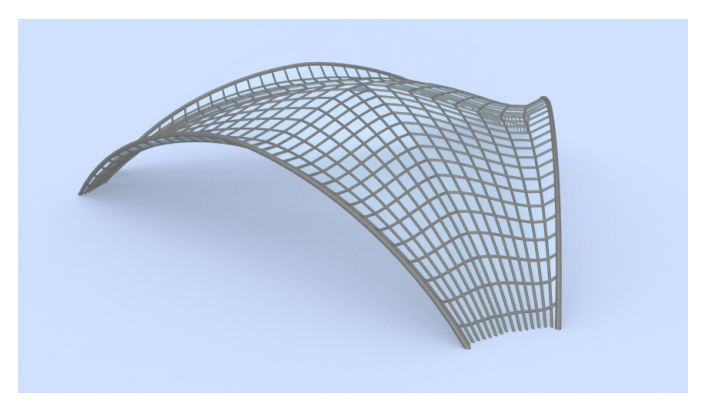

(b) The corresponding lifted mesh

Figure 4. A Marionette Meshes with a singularity.

For an arbitrary quad-mesh, it is possible to compute the number of guide curves that can be used to generate a Marionette Mesh. The mesh can be decomposed into simple quad domains without any singularity, for example by using the methods described in Tarini et al. (2011) or Takayama et al. (2013). For example, Figure 4a has six domains, the mesh in Figure 5a has nine domains. These domains are four sided, and it is possible to extract independent families of strip-domains, like displayed in Figure 5. Depending on the n-colorability of the mesh, the number of families varies. The example showed is two-colorable. As a result, two families of strips can be found and are shown in Figure $5 \mathrm{~b}$ and $5 \mathrm{c}$. Exactly one curve can be chosen across each strip-domain. Since strips are independent, the height of these nine curves can be chosen independently and will not over-constrain the problem. 


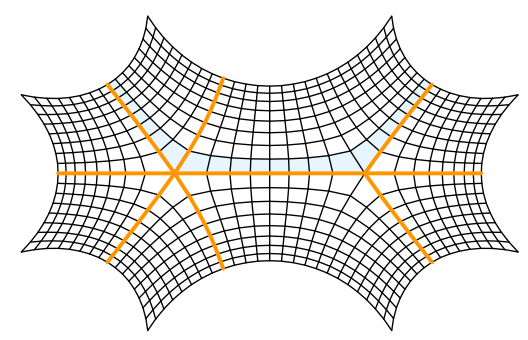

(a) Initial mesh

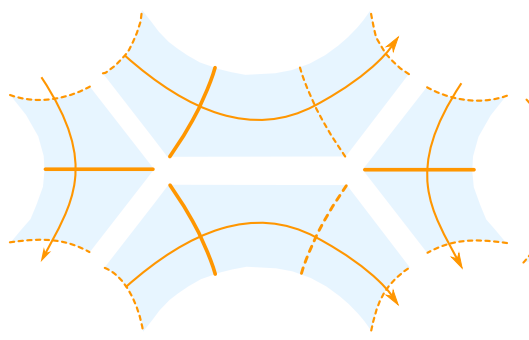

(b) Family of four strip-domains

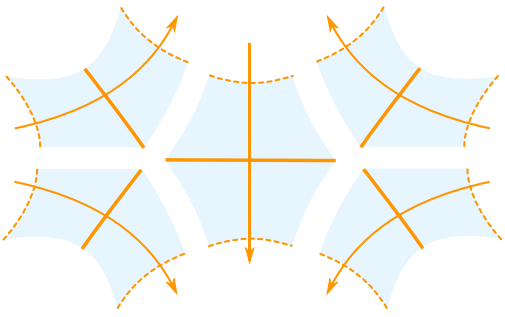

(c) Family of five strip-domains

Figure 5. Decomposition of a mesh into 2 families of strip-domains. Marionette Meshes can be generated by choosing one guide curve across each strip-domain.

\subsection{Closed Marionette Meshes}

Closed strips Marionette Meshes create PQ-meshes by propagation of a planarity constraint along strips. One can easily figure that if the strip is closed, the problem becomes over-constrained. Indeed, consider Figure 6: the plane view of a closed strip and the height of the points $\left(P_{i}\right)$ of one polyline are prescribed. If the height of the first point used for the propagation $P_{0}^{*}$ is chosen, the planarity constraint can be propagated along the strip. The points of the outer line are therefore imposed by the method, and the designer has no control on them. The last point $P_{N}^{*}$ is therefore generally different from the initial point $P_{0}^{*}$, leading to a geometrical incompatibility of PQ-meshes.

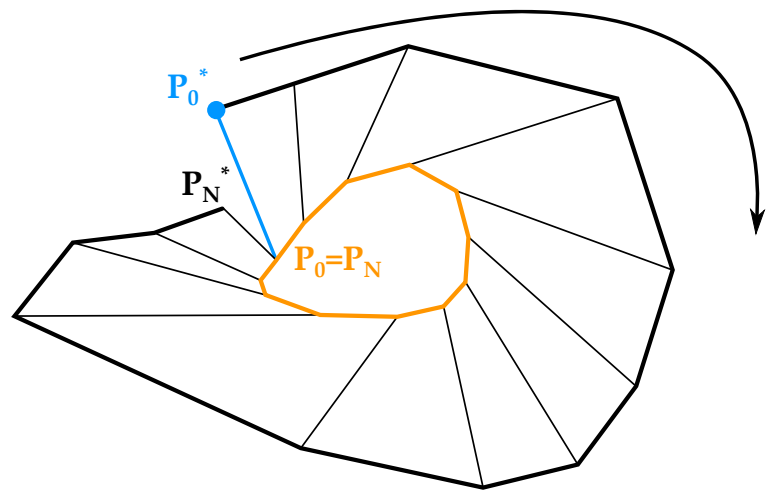

Figure 6. Closed Marionette Strip with incompatible closing condition induced by the prescription of the plane view of the whole strip (yellow) and the heights of the inner curve (blue).

In the following, we develop a method to deal with the geometrical compatibility of closed strips. The results however can then be extended to general Marionette Mesh with closed strips. Suppose that the two prescribed curves are defined as the inner closed curve and one radial curve (see Figure 6). By propagation of equation (2), we easily see that the height of the last point $z_{N}^{*}$ depends linearly on the height of the first point $z_{0}^{*}$ and on the heights of the points on the inner curve $\mathbf{Z}$. It also depends on the in-plane projection of the strip. Formally, there exists a vector $\mathbf{V}$ and a scalar $a$, both functions of the plane view so that:

$$
\mathbf{V} \cdot \mathbf{Z}+a \cdot z_{0}^{*}=z_{N}^{*}
$$

We are interested in the case where $z_{0}^{*}=z_{N}^{*}$. There are two possibilities:

1. $a=1$ : in this case, the condition restricts to $\mathbf{V} \cdot \mathbf{Z}=$ 0 and does not depend on $z_{0}^{*}$. The vector $\mathbf{Z}$ is in the hyperplane of $\mathbf{V}$, which leaves $N-1$ degrees of freedom.

2. $a \neq 1$ : there is only one solution for $z_{0}^{*}$. This is the most constrained case: the designer can only control the inner curve of the strip.

Detailed calculations on closed strips and particular examples satisfying the condition $a=1$ are given in Appendix A.

Closed meshes The meshes with one solution are less flexible, but they can still generate interesting shapes, like the one displayed on Figure 7, which recalls the example of Figure 6. The designer has a total control on the height of the inner curve and the plane view, but cannot manipulate freely the outer curve.

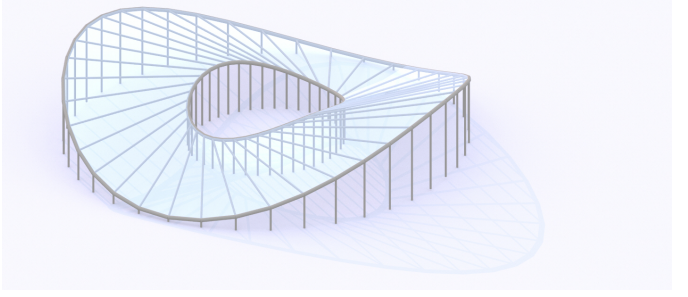

Figure 7. Architectural design with a closed Marionette Mesh, the height of the inner curve is prescribed, the designer does not have control on the outer curve.

The most interesting case occurs when the designer has potentially the control of two curves. It relies on a condition on the planar projection explained above. A simple case where this condition is fulfilled is when it has a symmetry. 
In this case, there is a $N-1$ parameters family of solutions for the height of the inner curve. The elevation of a closed guide curve can be chosen arbitrarely and projected into the hyperplane of normal $\mathbf{V}$, keeping the notations of equation (8). This operation is straight forward and allows to control the elevation of a second curve, like for open meshes. An example of this strategy is displayed in Figure 8, where all the meshes have the same planar projection, which has a plane of symmetry. The marionette mesh on the second row, and the column on the right in Figure 8 has however no plane of symmetry.

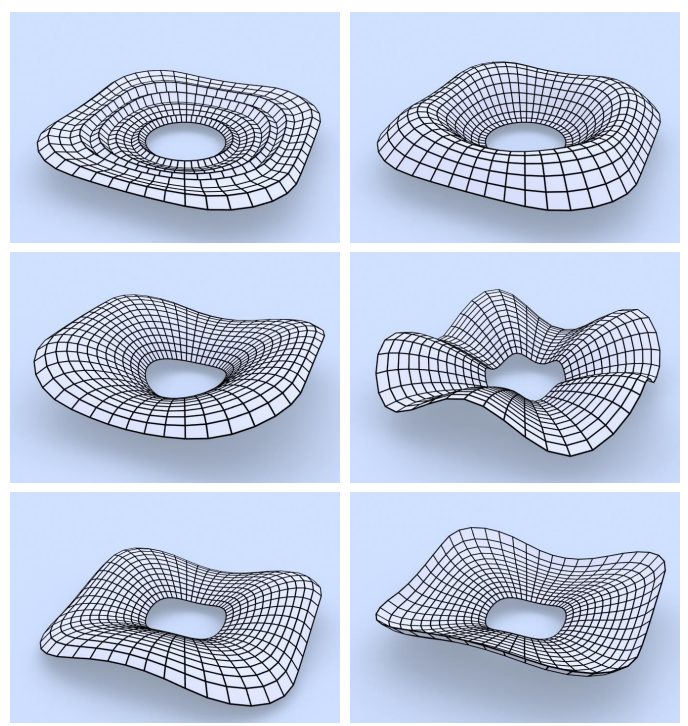

Figure 8. Some shapes with planar faces and a closed mesh generated with the method proposed in this paper. All the shapes are built from the same planar projection

Another look at the problem The problems specific to closed strips or meshes can be understood by the consideration of the equivalent smooth problem. The partial differential equation (5) remains unchanged but the boundary conditions expressed by equation (7) are not valid anymore. Indeed, a closed surface imposes a periodicity of the solution. Consider the case where we want the curves ( $u=$ constant) to be closed, there exist a certain period $T$ so that:

$$
\left\{\begin{array}{rrr}
f^{z}\left(u=u_{0}, v\right) & = & f_{1}(v) \\
\frac{\partial f^{z}}{\partial u}\left(u, v=v_{0}\right) & = & f_{3}(u) \\
\forall u, f^{z}(u, v+T) & = & f^{z}(u, v)
\end{array}\right.
$$

This additional boundary condition might over-constrain the problem and the existence of a solution is not certain.

\section{Architectural design with Marionette Meshes}

\subsection{Computational set-up}

The algorithms described in this paper have been implemented in the visual-scripting plug-in Grasshopper ${ }^{\mathrm{TM}}$ for the modelling software Rhino ${ }^{\mathrm{TM}}$. This allows interaction with other numerical tools necessary for architectural design, like finite-element analysis software Karamba ${ }^{\mathrm{TM}}$. An example of interaction between fabrication-aware shape generation and structural analysis is shown in section 3.4.

Marionette Meshes only require the solution of a sparse linear system. The computation time is thus low, typically it takes $3 \mathrm{~ms}$ to lift a mesh of 10,000 faces, with no pre-factorisation involved. Real-time computation provides great design flexibility, even for large meshes. It also opens possibilities to perform optimisation with numerous function calls, for example with genetic algorithms, as discussed in Section 3.4.

The basic input of the method are a planar projection and several elevation curves. The marionette framework allows the architect and the engineer to choose a topology and some mesh features (like alignment of the mesh to a boundary) and generates a design space of feasible solutions. In comparison, post-rationalisation techniques fit perfectly a target geometry (for example with conjugate fields integration), with less control over the grid topology. Since parametrisation is equally important as shape in the overall æsthetics and structural behaviour of a grid structure, the marionette technique,like some optimisation-based algorithms (Tang et al. 2014; Deng et al. 2015), offers an interesting alternative to post-rationalisation.

In our framework, the planar projections are generated with NURBS patches, and the elevation curves are drawn as Bézier curves. The smoothness of the final mesh depends thus on the smoothness of the in-plane parametrisation. A $C^{0}$ projection yields a $C^{0}$ solution to the hyperbolic equation (4), so that shape functions with creases can easily be propagated through the mesh (Mesnil et al. 2017a). Figure 9 shows a corrugated shape generated from a $C^{0}$ planar projection and smooth guide curves. Such corrugations can be used in folded plate structures, and could extend the formal possibilities of methods developed in (Robeller et al. 2015) or discussed in (Lebée 2015).

\subsection{Geometrical optimisation}

General remarks The method used in this paper constructs a space of solutions with planar facets. This space is a vector space, which has some interesting implications for 


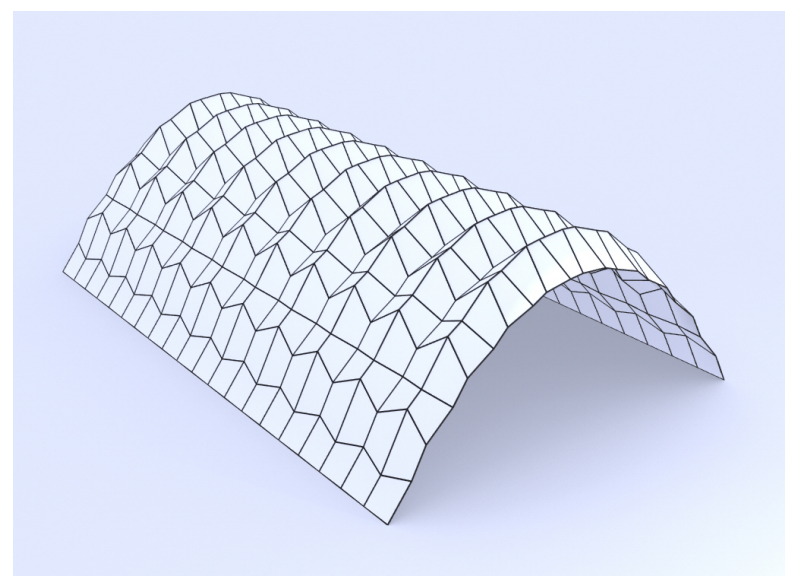

Figure 9. A non-smooth mesh with planar facets generated with the Marionette method.

some optimisation problems. We can indeed see the planarity constraint as a linear constraint on the coordinates of all the vertices of a mesh. There exists a matrix $\mathbf{A}$ and list of heights $\mathbf{z}$ and $\mathbf{z}_{\mathbf{p}}$ so that:

$$
\mathbf{A} \cdot \mathbf{z}_{\mathbf{p}}=\mathbf{z}
$$

The marionette method gives an intuitive way to construct this matrix, as $\mathbf{z}_{\mathbf{p}}$ corresponds to the heights of vertices on the guide curves and $\mathbf{A}$ depends on the planar projection. For a mesh with $N M$ faces, $\mathbf{A}$ is a matrix of size $(N+M+1,(N+1) \cdot(M+1))$

It is a well-known fact that minimisation of quadratic functions under linear constraints is equivalent to the solving of a linear system (Nocedal and Wright 2006). An example of such optimisation problems with useful applications for architectural design is given in the following.

Surface fitting A common problem described in the literature is the approximation of a given shape with a PQ-mesh. In the followings, we consider that the designer prescribes a planar projection and looks for the closest Marionette Mesh to a reference surface.

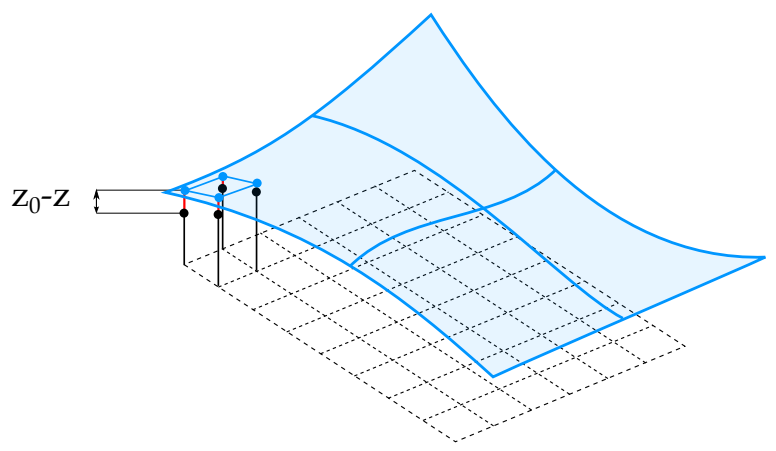

Figure 10. Optimisation problem: approximation of a reference surface with a given planar projection (dashed lines).
The problem is illustrated in Figure 10: the height of the vertices in the Marionette Mesh are written $\mathbf{z}$, the heights of the points on the reference surface are written $z_{0}$. The function to minimize is written as follows.

$$
J(\mathbf{z})=\left(\mathbf{z}-\mathbf{z}_{\mathbf{0}}\right)^{T}\left(\mathbf{z}-\mathbf{z}_{\mathbf{0}}\right)
$$

The design space is the Marionette Meshes which have the considered planar projection. This constraint is written in equation (10). The optimisation problem follows:

$$
\min _{\mathbf{z}=\mathbf{A} \mathbf{z}_{\mathbf{p}}} J(\mathbf{z})=\min \left(\mathbf{A} \mathbf{z}_{\mathbf{p}}-\mathbf{z}_{\mathbf{0}}\right)^{T}\left(\mathbf{A} \mathbf{z}_{\mathbf{p}}-\mathbf{z}_{\mathbf{0}}\right)
$$

Expanding the equation, one gets:

$$
J_{p}\left(\mathbf{z}_{\mathbf{p}}\right)=\mathbf{z}_{\mathbf{p}}{ }^{T} \mathbf{A}^{T} \mathbf{A} \mathbf{z}_{\mathbf{p}}-2 \mathbf{z}_{\mathbf{p}}^{\mathbf{T}} \mathbf{A}^{\mathbf{T}} \mathbf{z}_{\mathbf{0}}{ }^{T}+\mathbf{z}_{\mathbf{0}}{ }^{T} \mathbf{z}_{\mathbf{0}}
$$

A necessary condition to find a solution is to verify that $\nabla J_{p}=\mathbf{0}$. The system reduces therefore to:

$$
\mathbf{A}^{\mathbf{T}} \mathbf{A} \mathbf{z}_{\mathbf{p}}=\mathbf{A}^{\mathbf{T}} \mathbf{z}_{0}
$$

Equation (14) is typical of least square problem. It is clear that the rank of the matrix $\mathbf{A}$ is $(N+M+1)$. It follows that the rank of $\mathbf{A}^{\mathbf{T}} \mathbf{A}$ is also $(N+M+1)$. Since $\mathbf{A}^{\mathbf{T}} \mathbf{A} \in$ $\mathcal{M}(N+M+1, N+M+1)$, this matrix is invertible. Equation (14) has therefore one unique solution. Since $\mathbf{A}^{\mathbf{T}} \mathbf{A}$ is clearly definite positive, it follows that the extremum is in fact a local minimum. Finally, the behavior when $\left\|\mathbf{z}_{\mathbf{p}}\right\| \longrightarrow$ $\infty$ demonstrates that this is a global minimum.

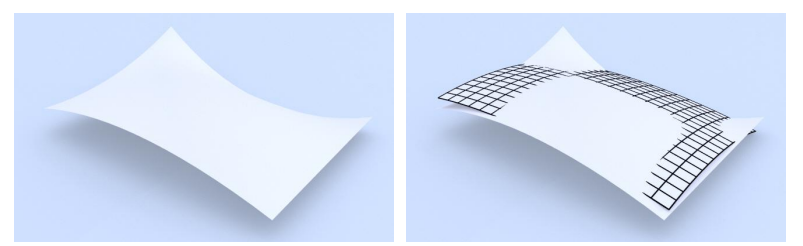

Figure 11. A target surface (left), and the optimal approximation by a surface of translation (right).

An application is illustrated in Figure 11, where a target NURBS is approached by a surface of translation, which are well-known in architectural design (Glymph et al. 2004). This optimal can be considered poor, but the key information is that it is the best in the design space chosen by the designer, so that the designer knows that to improve the solution, he has to explore other planar projections or mesh topologies. The surface displayed is indeed the best solution possible for the planar projection chosen by the designer. As computation are done in real-time, it is easy to generate very quickly different plane views with different topologies, keeping control of the aesthetic and layout of the cladding. 
The mesh topologies could be generated from a catalogue, like the ones generated in (Takayama et al. 2014) and their relevance for the shape-fitting problem could be efficiently assessed with the marionette method. The selection of a proper mesh topology remains however open for future work.

\subsection{Shape exploration with Marionette Meshes}

The framework introduced here intrinsically account for planarity of panels. Its mathematical formulation is however suited for many architectural constraints. Hard constraints must be fulfilled exactly, whereas soft constraints are included into the function to minimize (Nocedal and Wright 2006). Since the planarity constraint is linear, soft constraints expressed as linear or quadratic functions can easily be included in the objective function. In this case, the optimisation problem will be similar to a classical least square problem and can be solved efficiently.

Hard constraints defined by linear equations are treated effectively within the proposed framework. Examples of linear constraints are prescribed volume or a maximal allowable height. The marionette method imposes $N M-$ $(N+M-1)$ out of $N M$ parameters, this means that another $N+M-1$ linear constraints can be applied without over-constraining the optimisation problem.

Perhaps the most interesting application is the prescription of a boundary, as depicted in Figure 12. In this figure, the planar projection is imposed and the user prescribes the height of some points of the mesh along a curve (white circles). In this case, the number of prescribed points is superior to the number of degrees of freedom, and the problem might be overconstrained. It might hence be preferable to turn this problem into a soft constrained problem with a quadratic function to minimize.

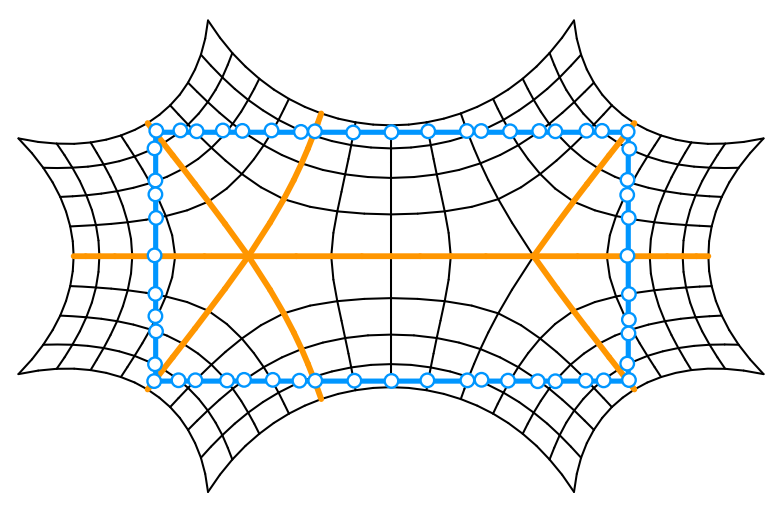

Figure 12. A plane view (thin lines) with a prescribed boundary (thick lines).

Other constraints could be used. For example, in the manner of NURBS-modelling, the user could control the height of some handle-points, each handle decreasing the size of the space of solution by 1 dof. This kind of approach has been used in optimisation-based shape exploration, but it looses the notion of global shape control.

\subsection{Case study: fabrication-aware structural optimisation}

The formal possibilities offered by Marionette Meshes are broad enough to offer an interesting design space for engineering problems. Among them, structural optimisation is a particularly relevant. The quick generation of a parameterised design space and the coupling with advanced analysis software seems particularly promising (Preisinger and Heimrath 2014). Indeed, non-linear criteria, like the buckling capacity, are of high importance for practical design of thin shell or grid shells (Firl and Bletzinger 2012).

An illustration of the potential of Marionette Meshes for a structurally-informed architectural design is proposed in Figure 13: the shell is a Marionette mesh spanning over an ellipse. The plane view is inspired by Figure 1. The mesh is consituted of six NURBS patches and has two singularities (white dots in the image), guide curves are found with the method proposed in this paper. The boundary curve is constrained in the horizontal plane (blue curve on Figure 13). One curve in the other direction (orange curve in Figure 13) defines the whole elevation of the dome. The shell is submitted to gravity load. All the translations at the outer boundary are restricted and rotations at the supports are allowed (hinges). The model is computed with Finite Element software Karamba3D ${ }^{\mathrm{TM}}$. The shape generation of a 1000 faces mesh requires less than $1 \mathrm{~ms}$ with the Marionette technique, far less than the assembly and computation of a shell model with FEM.

The structure is optimised towards a minimum of the total elastic energy by the means of genetic algorithms. In Figure 13, the color scheme is used to represent the maximal principal stress $\sigma_{11}$ on the upper side of the shell. Area with tensile stress should appear in blue, but are not visible on the Figure. Indeed, tensile stress in Figure 13 are almost nonexistent: the maximal tensile stress is of . $2 \mathrm{MPa}$ for a dome with a span of $40 \mathrm{~m}$ and a shell thickness of $10 \mathrm{~cm}$. Hence, if defined properly with an accurate number of singularities, the design space offered by Marionette Meshes is wide enough to find compression-dominant shapes by the means of structural optimisation. 

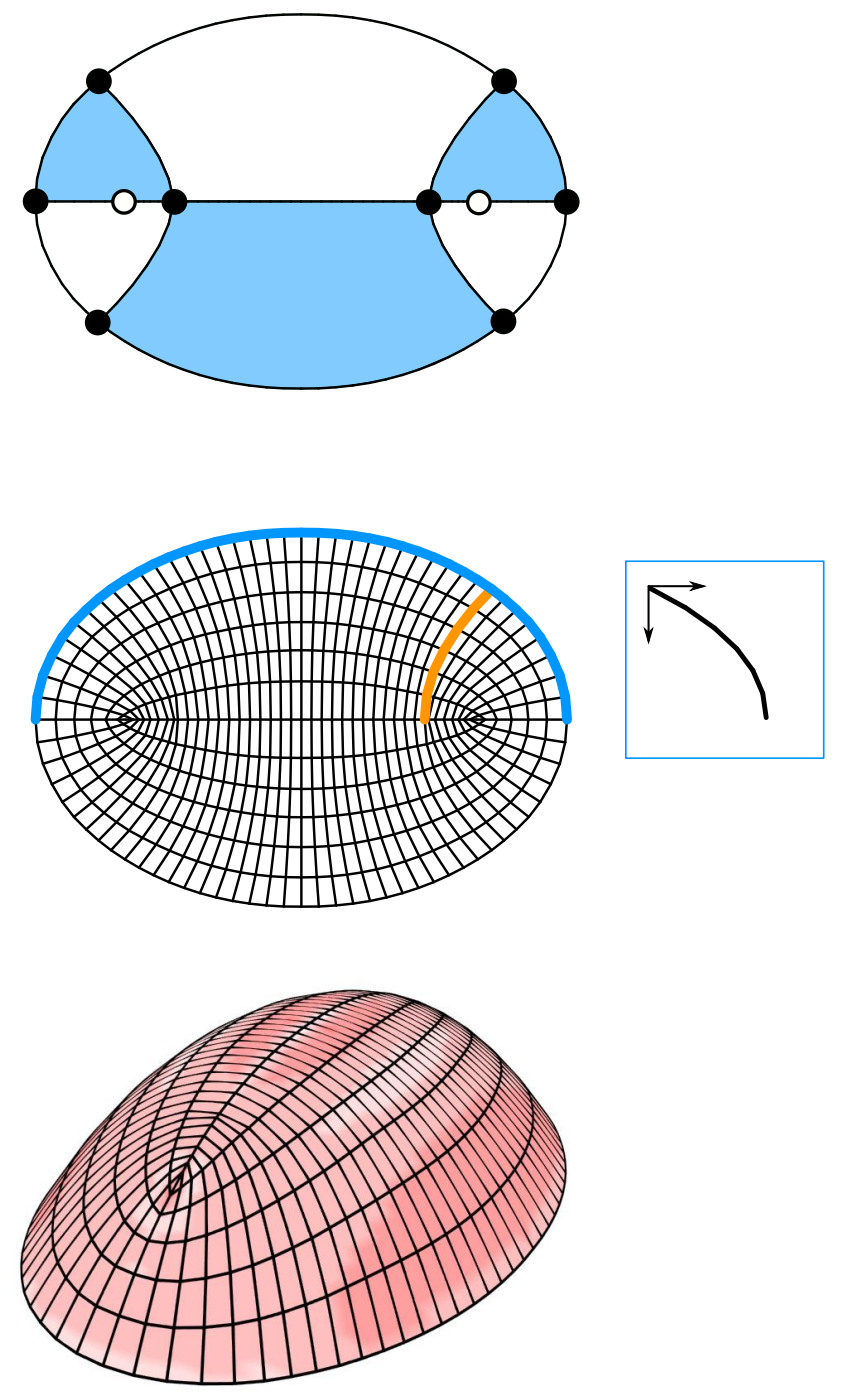

Figure 13. A result of an optimisation procedure: the shell structure is a Marionette Mesh constructed from six patches (top) minimising total elastic energy. Middle: guide curves and top view. Bottom: red areas indicate compression, the shell works mostly with compression.

\section{Generalisation of the method}

\subsection{General projections}

It appeared that prescribing a horizontal view and applying the propagation technique presented here only allows for the modelling of height fields. This is a limitation of this method, although height fields surfaces are commonly used for roof covering. Other projections can be used for more shape flexibility. The planarity constraint for a quad can be extendend to the case of non-parallel projections, like in Figure 14.

Some projections are of practical interest for archetypal projects. Towers and facades can be modelled with cylindrical projections. Stadia can be designed using projections on torus or on moulding surfaces, the offset

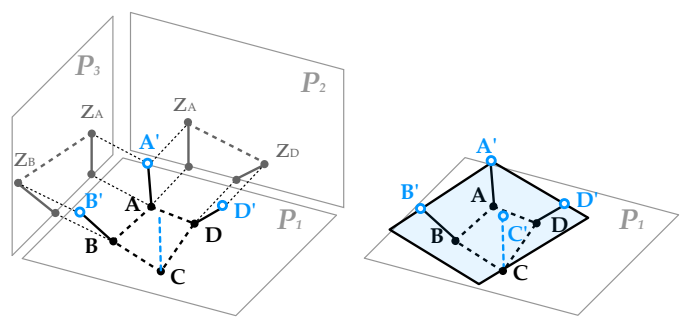

Figure 14. A Marionette Quad with non-parallel guide lines.

directions corresponding to the normals of the smooth surface. Moulding surfaces fit naturally the geometry of stadia (see Figure 15a) and have some interesting features, discussed in (Mesnil et al. 2015):

- Their natural mesh contains planar curves, which are geodesics of the surface: the planarity is preserved by the marionette transformation.

- They are naturally meshed by their lines of curvatures, which gives a torsion-free beam layout on the initial surface, and on the final shape.

\subsection{Extension to other patterns}

This section proposes to extend the Marionette method to other patterns than quads. First, we discuss the estimation of the size of the design space offered by the marionette technique in the most general cases. We illustrate then those remarks on the generality of the method with various patterns.

Size of the design space The facet planarity constraint is linear, which means that the space of meshes with planar facets is a vector space. (Deng et al. 2013) proposed a criterion to evaluate the dimension of this vector space. For each facet, three points can be chosen independently (3 d.o.f for each points) and the remaining points must be chosen in the constructed plane (1 d.o.F deleted for these nodes). Writing $n_{F}$ the number of vertices for each face, the estimation of the size of the space of meshes with planar facets follows (Deng et al. 2013):

$$
N \sim 3 N_{\text {nodes }}-\sum_{\text {Faces }}\left(n_{F}-3\right)
$$

For a quad mesh, we get $N \sim 2 N_{\text {nodes }}$. This number is high and is difficult to interpret for the designer. The projection technique used in this paper reduces the size of the design space. Since the planar projection is prescribed, each point looses 2 d.o.f. Equation (15) writes: 

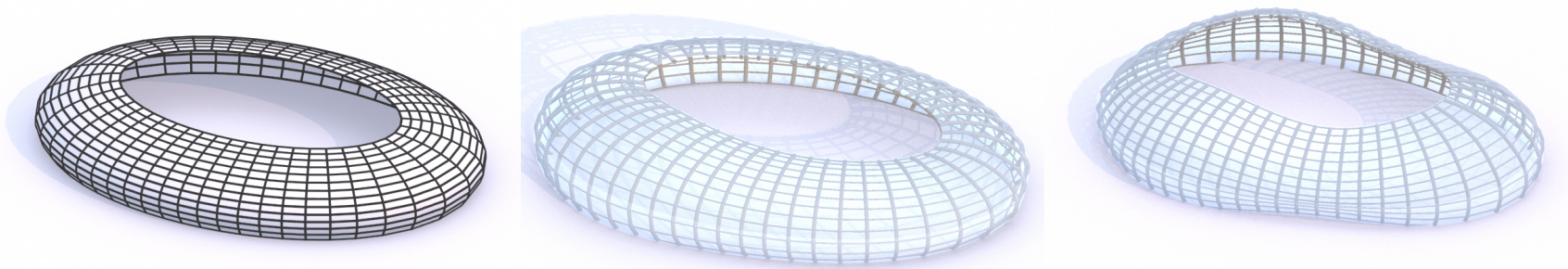

(a) Reference moulding surface

(b) Non-symmetrical design

(c) Symmetrical design

Figure 15. Design of stadia obtained from a projection on a moulding surface: the prescribed curves are the inner ring and a section curve.

$$
N_{\text {marionette }} \sim N_{\text {nodes }}-\sum_{\text {Faces }}\left(n_{F}-3\right)
$$

The size of the design space is reduced compared to general methods, but the smoothness of the final shape is easily controlled. Note that techniques relying on generation of the whole vector space have to introduce fairing energies, as the design space contains both smooth and non-smooth meshes.

For a quadrilateral mesh with $n \times m$ faces without singularity, we have $N_{\text {nodes }}=(n+1) \cdot(m+1)$, and $n m$ faces. The application of equation (16) shows that the size of the design space is $n+m+1$, which is exactly what is found by the marionette method.

Application to non-standard patterns Equation (16) can be applied to meshes composed of triangles and hexagons, also known as Kagome lattices. It reveals that the number of d.o.f is comparable to the one of quadrilateral meshes. There is therefore a straight forward way to lift Kagome lattices with the marionette technique. Figure 16a shows the guide curves for the Kagome pattern. Other isolated points are required to lift the mesh. The height of these points can for example be chosen in order to optimise mesh fairness, which has been characterised in numerous works by an energy $\mathcal{F}$ defined in equation (17), where $\mathbf{v}_{\mathbf{i}}$ is the $i^{\text {th }}$ vertex of a polyline:

$$
\mathcal{F}=\sum_{\text {polylines }} \sum_{i}\left\|\mathbf{v}_{\mathbf{i}}-2 \mathbf{v}_{\mathbf{i}+\mathbf{1}}+\mathbf{v}_{\mathbf{i}+\mathbf{2}}\right\|^{2}
$$

The functional is quadratic and is not difficult to minimise under linear constraints. Figure $16 \mathrm{c}$ shows a mesh derived from an hexagonal pattern: three guide curves can be used to lift the mesh. The number of degrees of freedom of the examples of Figure 16 are evaluated in Appendix B.

Illustration Figure 17 shows a Kagome lattice covered with planar facets generated with the marionette method. The design started from a planar projection generated with a NURBS patch, a Kagome was then generated following the isoparametric lines and lifted with the marionette technique. One of the guide curve is the parabolic arch of the entrance, the other is an undulating curve following the tunnel. Like for PQ-meshes, the computation is done in real-time.

\section{Conclusion}

We have introduced an intuitive technique for interactive shape modelling with planar facets. It is based on descriptive geometry, which has been used by architects and engineers for centuries. The concept has many applications, in particular the modelling of PQ-meshes with or without singularity. Some examples show the formal potential of our method. The framework was also extended to Kagome and dual-Kagome lattices. It is likely that other polyhedral patterns can be treated with the Marionette technique. The generality of the method has also been demonstrated by changing the projection direction, a method with large potential if used on mesh with remarkable offset properties.

Our discussion on marionette meshes with singularities highlights the fact that the choice of mesh topology influences greatly the size and nature of the design space for meshes with planar facets. Selecting a proper mesh topology is thus crucial in practice. This article dealt with the definition and implementation of the marionette method as a parametric design space exploration. The choice of a relevant mesh topology a priori from boundary conditions should be addressed in future work.

Quadratic optimisation problems, like surface-fitting problems can be solved efficiently with the marionette technique. A simple example where only the heights of the guide curve are the only parameters was detailed, but controlling the plane view with NURBS patches could allow for a more general solution of such problems. The 


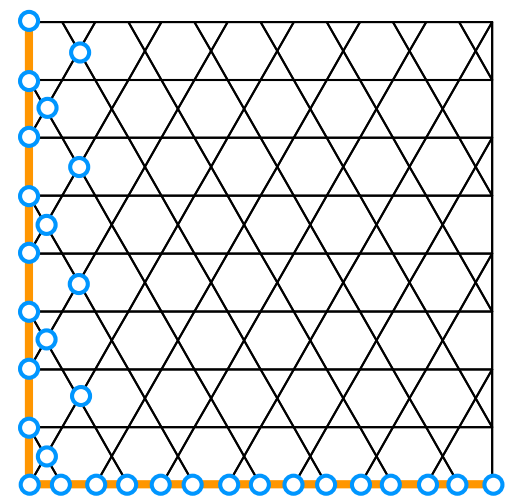

(a) Kagome lattice

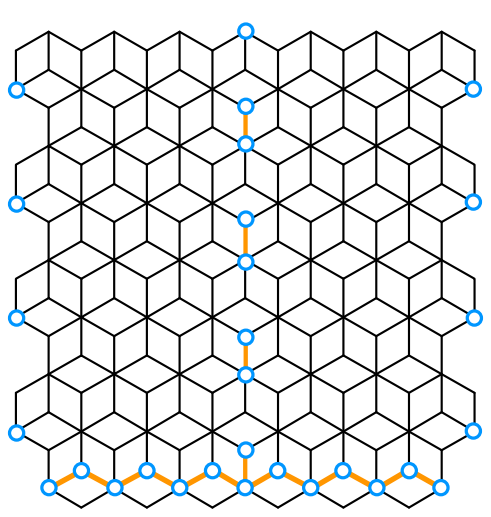

(b) Dual Kagome lattice

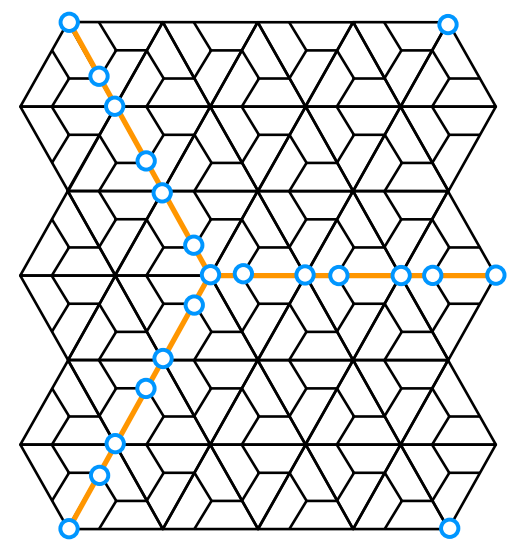

(c) Hex pattern

Figure 16. Marionette method applied to several patterns, white dots correspond to prescribed heights.

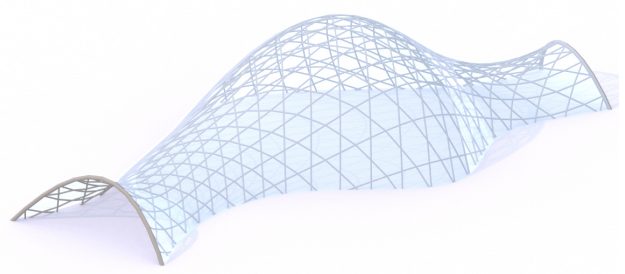

Figure 17. Free-form design covered by planar Kagome lattice.

separation of variables in horizontal plane and vertical plane can potentially give birth to efficient numerical methods for geometrical optimisation.

Furthermore, we made a comment on the underlying smooth problem solved by the method, which gives indications on the smoothness of the shapes arising form this framework. We have seen that this smoothness depends on the smoothness of both the planar projection and the guide curves, which can be generated with any usual modelling tool based on NURBS, T-spline and Bézier curves. Moreover, it was shown that marionette meshes give an intuitive illustration on the principle of subspace exploration, a powerful tool for constrained optimisation of meshes. It was shown in Section 3.4 that the marionette method could be used as an alternative to NURBS modelling for the parametrisation of structural optimisation problems for thin shells or gridshells. The relative performances of the two modelling technique in the context of structural optimisation should be assessed in future work.

\section{Acknowledgements}

This work was made during Mr. Mesnil doctorate within the framework of an industrial agreement for training through research (CIFRE number 2013/1266) jointly financed by the company
Bouygues Construction SA, and the National Association for Research and Technology (ANRT) of France.

\section{References}

Adriaenssens S, Ney L, Bodarwe E and Williams C (2012) Finding the form of an irregular meshed steel and glass shell based on construction constraints. Journal of Architectural Engineering 18(3): 206-213.

Alinhac S (2009) Hyperbolic partial differential equations. Springer Science \& Business Media.

Bagneris M, Motro R, Maurin B and Pauli N (2008) Structural Morphology issues in Conceptual Design of Double Curved Systems. International Journal of Space Structures 23(2): 79$-87$.

Block P and Ochsendorf J (2007) Thrust network analysis: A new methodology for three-dimensional equilibrium. Journal of the International Association for Shell and Spatial Structures 48: 167-173.

Bobenko A and Huhnen-Venedey E (2012) Curvature line parametrized surfaces and orthogonal coordinate systems: discretization with Dupin cyclides. Geometriae Dedicata 159(1): 207-237.

Bobenko A and Suris YB (2008) Discrete Differential Geometry: Integrable Structure. American Mathematical Society. URL http://bookstore.ams.org/gsm-98.

Bouaziz S, Deuss M, Schwartzburg Y, Weise T and Pauly M (2012) Shape-Up: Shaping Discrete Geometry with Projections. Computer Graphics Forum 31(5): 1657-1667. DOI:10.1111/ j.1467-8659.2012.03171.x. URL http://doi.wiley. com/10.1111/j.1467-8659.2012.03171.x.

Deng B, Bouaziz S, Deuss M, Kaspar A, Schwartzburg Y and Pauly M (2015) Interactive design exploration for constrained meshes. Computer-Aided Design 61: 13-23. DOI:10.1016/j. 
cad.2014.01.004. URL http://www.sciencedirect. com/science/article/pii/s0010448514000050.

Deng B, Bouaziz S, Deuss M, Zhang J, Schwartzburg Y and Pauly M (2013) Exploring local modifications for constrained meshes. Computer Graphics Forum 32(2 PART1). DOI: 10.1111/cgf.12021.

Firl M and Bletzinger KU (2012) Shape optimization of thin walled structures governed by geometrically nonlinear mechanics. Computer Methods in Applied Mechanics and Engineering 237-240: 107-117. DOI:10.1016/j.cma.2012. 05.016. URL http://linkinghub.elsevier.com/ retrieve/pii/s0045782512001727.

Glymph J, Shelden D, Ceccato C, Mussel J and Schober H (2004) A parametric strategy for free-form glass structures using quadrilateral planar facets. Automation in Construction 13(2): 187-202. DOI:10.1016/j.autcon.2003. 09.008. URL http://linkinghub.elsevier.com/ retrieve/pii/s0926580503001055.

Javary A (1881) Traité de géométrie descriptive. Delagrave. URL http://gallica.bnf.fr/ark:/12148/ bpt 6k28506r.

Jiang C, Tang C, Tomicic M, Pottmann H and Wallner J (2014) Interactive Modeling of Architectural Freeform Structures Combining Geometry with Fabrication and Statics. In: Block P, Wang W and Knippers J (eds.) Advances in Architectural Geometry. Springer.

Klein F (1893) Vergleichende Betrachtungen über neuere geometrische Forschungen. Mathematische Annalen 43(1): 63-100. URL http://link. springer.com/10.1007/BF01446615http:

//download.springer.com/static/pdf/

801/art:10.1007/BF01446615.pdf?

originUrl=http://link.springer.com/

article/10.1007/BF01446615\{\&\}token2=

$\exp =1452526797\{\sim$ acl=/static/pdf / $801 /$

$\operatorname{art}\{\div\} 3 \mathrm{~A} 10.1007\{\%\}$ 2FBF 01446615 .p.

Lebée A (2015) From Folds to Structures, a Review. International Journal of Space Structures 30(2): 55-74.

Leroy C (1857) Traité de stéréotomie, comprenant les applications de la géométrie descriptive à la théorie des ombres, la perspective linéaire la gnomonique, la coupe des pierres et la charpente, avec un atlas composé de 74 planches in folio. Mallet-Bachelier.

Liu Y, Wang W, Pottmann H, Wallner J and Yong-Liang Y (2006) Geometric Modeling with Conical Meshes and Developable Surfaces. ACM Transactions on Graphics 25(3): 681-689.

Mesnil R, Douthe C, Baverel O, Caron JF and Leger B (2017a) Structural morphology and performance of plated structures with planar quadrilateral facets. Journal of the International Association for Shell and Spatial Structures 58(1).

Mesnil R, Douthe C, Baverel O and Leger B (2017b) Generalised cyclidic nets for shape modelling in architecture. International Journal of Architectural Computing 15(2).

Mesnil R, Douthe C, Baverel O, Léger B and Caron JF (2015) Isogonal moulding surfaces: a family of shapes for high node congruence in free-form structures. Automation in Construction 59: 38-47.

Miki M, Igarashi T and Block P (2015) Parametric Self-supporting Surfaces via Direct Computation of Airy Stress Functions. ACM Transaction on Graphics 34(4). URL http: // doi. acm.org/10.1145/2766888.

Monge G (1798) Géométrie descriptive. Baudouin.

Nocedal J and Wright S (2006) Numerical Optimization. Springer Science \& Business Media.

Pottmann H, Asperl A, Hofer M and Kilian A (2007) Architectural Geometry. Bentley Institute Press. ISBN 978-1-934493-04-5.

Preisinger C and Heimrath M (2014) Karamba - A Toolkit for Parametric Structural Design. Structural Engineering International 24(2): 217-221. DOI: 10.2749/101686614X13830790993483. URL http: //openurl.ingenta.com/content/xref?genre= article\&issn=1016-8664\&volume $=24 \&$ issue $=2 \&$ spage $=217$.

Rippmann M, Lachauer L and Block P (2012) Interactive Vault Design. International Journal of Space Structures 27(4): 219230.

Robeller C, Stitic A, Mayencourt P and Weinand Y (2015) Interlocking folded plate: Integrated mechanical attachment for structural wood panels. In: Advances in Architectural Geometry 2014. Springer, pp. 281-294.

Schober H (2002) Geometrie-prinzipien für wirtschaftliche und effiziente schalentragwerke (teil 1). Bautechnik 79(1): 16-24.

Takayama K, Panozzo D, Sorkine-Hornung A and SorkineHornung O (2013) Sketch-Based Generation and Editing of Quad Meshes. ACM Transactions on Graphics 32(4): 97.

Takayama K, Panozzo D and Sorkine-Hornung O (2014) Patternbased quadrangulation for $\mathrm{n}$-sided patches. In: Computer Graphics Forum, volume 33. Wiley Online Library, pp. 177184.

Tang C, Sun X, Gomes A, Wallner J and Pottmann H (2014) Form-finding with Polyhedral Meshes Made Simple. ACM Transactions on Graphics 33(4).

Tarini M, Puppo E, Panozzo D, Pietroni $\mathrm{N}$ and Cignoni $\mathrm{P}$ (2011) Simple Quad Domains for Field Aligned Mesh Parametrization. ACM Transactions on Graphics 30(6): 142. 
Yang Yl, Yang YJ, Pottmann H and Mitra NJ (2011) Shape Space Exploration of Constrained Meshes. ACM Transactions on Graphics 30: 124.

\section{A Some results on closed strips}

The aim of this section is to discuss with more detail the problem of closed strips. First, we write the propagation problem on a strip. This step is purely computational, but is necessary to introduce a quantity of interest. We interpret then the geometrical meaning of the compatibility condition with respect to the mathematical formalism introduced. Then, we present some particular cases where the closing of a strip is possible regardless of the choice of the height on the outer curve.

\section{A.1 Propagation equation}

Consider the closed strip discussed in Section 2.5. For each facet, we can apply the planarity constraint of equation (2). Writing $z_{i}$ the height of the $i^{\text {th }}$ point of the inner curve and $z_{i}^{*}$ the height of the $i^{\text {th }}$ point of the outer curve, we can rewrite this equation. For the sake of simplicity, we replace the ratios of the $2 \mathrm{D}$ determinants by scalars $a_{i}$ and $b_{i}$. We make following identifications:

$$
\left\{\begin{array}{rrr}
P_{i} \Leftrightarrow & A \\
P_{i+1} \Leftrightarrow & B \\
P_{i}^{*} \Leftrightarrow & C \\
P_{i+1}^{*} \Leftrightarrow & D \\
a_{i} \Leftrightarrow & \frac{\operatorname{det}_{2 \mathrm{D}}(\mathbf{A B}, \mathbf{A C})}{\operatorname{det}_{2 \mathrm{D}}(\mathbf{A B}, \mathbf{A D})} \\
b_{i} \Leftrightarrow & \frac{\operatorname{det}_{2 \mathrm{D}}(\mathbf{A D}, \mathbf{A C})}{\operatorname{det}_{2 \mathrm{D}}(\mathbf{A B}, \mathbf{A D})}
\end{array}\right.
$$

We get hence following equation:

$$
z_{i+1}^{*}=\left(1-a_{i}-b_{i}\right) z_{i}+a_{i} z_{i}^{*}+b_{i} z_{i+1}
$$

We make the following hypothesis, which is easily verified by recurrence:

$$
\forall i>0, z_{i}^{*}+=\sum_{k=0}^{i} v_{k} z_{k}+A_{i} z_{0}^{*}
$$

In fact, we can be even more precise and compute the value of $A_{i}$. We make the hypothesis that:

$$
\forall i>0, A_{i}=\prod_{k=0}^{i-1} a_{k}
$$

Proof This is true for $i=1$ due to equation (18). Then, we proceed by recurrence. Assume that (20) is true for $i$, then we show that this is true for $i+1$. We plug equation (19) into equation (18) and get:

$$
z_{i+1}^{*}+=\left(1-a_{i}-b_{i}\right) z_{i}+a_{i}\left(\sum_{k=0}^{i} v_{k} z_{k}+A_{i} z_{0}^{*}\right)+b_{i} z_{i+1}
$$

There is only one term in $z_{0}^{*}$, and it verifies equation (20).

\section{A.2 Geometrical interpretation}

The ratios $a_{i}$ can be interpreted with elementary plane geometry. Consider Figure 18: the ratio $a$ is defined with 2D determinants and can be expressed with the vectors norms and angles. We have:

$$
a=\frac{\|\mathbf{A B}\|\|\mathbf{A} \mathbf{C}\| \sin \beta}{\|\mathbf{A B}\|\|\mathbf{A D}\| \sin \alpha}
$$

We recognise the areas of the triangles $A B C$ and $A B D$, so that $a$ can be rewritten as:

$$
a=\frac{\mathcal{A}_{A B C}}{\mathcal{A}_{A B D}}
$$

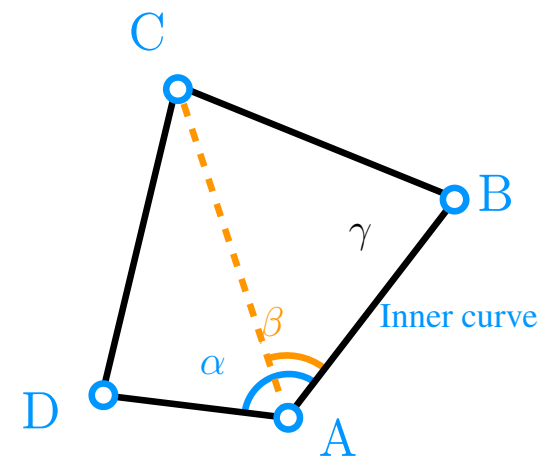

Figure 18. Planar projection of a quadrilateral and angle notations

The two triangles used for the computation of $a_{i}$ are shown in Figure 19.
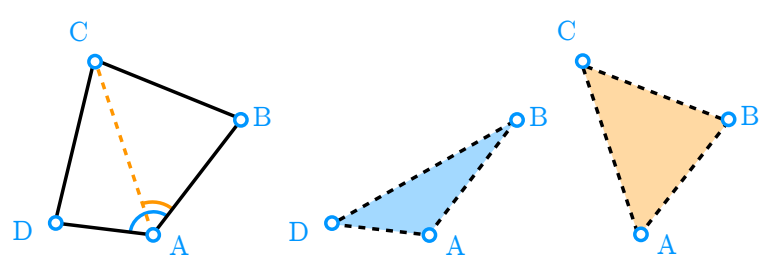

Figure 19. The two triangles used to compute $a_{i}$. 


\section{A.3 General solutions for a closed strip}

Recall that we are interested in finding the solutions so that $z_{N}^{*}=z_{0}^{*}$, which also writes:

$$
z_{N}^{*}-z_{0}^{*}=\sum_{k=0}^{N} v_{k} z_{k}+\left(\prod_{k=0}^{N-1} a_{k}-1\right) z_{0}^{*}=0
$$

We also want the space of solutions to be as large as possible, and therefore, we don't want it to depend on the choice of the height on the outer curve $z_{0}^{*}$. This implies a new equation:

$$
\prod_{k=0}^{N-1} a_{k}=1
$$

In the following, we discuss the invariance of this equation under some transformations and show some particular cases where it is satisfied.

\section{A.4 Invariance}

To have a complete overview on the problem of closed strips, we provide transformations that map compatible strips to other compatible strips. The study of group of transformations that preserve a given quantity is at the core of modern geometry, and for the sake of completeness, we show this point of view dating back from Felix Klein (Klein 1893).

Linear maps The transformations we are interested in preserve equation (24). The most straight forward way to do this is to preserve each $a_{i}$. It is clear that all linear transformation in the plane (translation, scaling, shearing) preserve each individual ratio. Consider indeed transformations defined by:

$$
f(x, y)=\left(\begin{array}{ll}
m_{11} & m_{12} \\
m_{21} & m_{22}
\end{array}\right) \cdot\left(\begin{array}{l}
x \\
y
\end{array}\right)+\left(\begin{array}{c}
X_{0} \\
Y_{0}
\end{array}\right)
$$

Consider two points $\left(x_{0}, y_{0}\right)$ and $\left(x_{1}, y_{1}\right)$. We write their image by $f$ respectively $\left(x_{0}^{\prime}, y_{0}^{\prime}\right)$ and $\left(x_{1}^{\prime}, y_{1}^{\prime}\right)$. We call $\mathbf{M}$ the matrix written in equation (25), then we have:

$$
\operatorname{det}\left(\begin{array}{ll}
x_{0}^{\prime} & x_{1}^{\prime} \\
y_{0}^{\prime} & y_{1}^{\prime}
\end{array}\right)=(\operatorname{det} \mathbf{M})^{2} \operatorname{det}\left(\begin{array}{ll}
x_{0} & x_{1} \\
y_{0} & y_{1}
\end{array}\right)
$$

It is clear that the linear map preserves the ratio of $2 \mathrm{D}$ determinant, since the factor $\operatorname{det} \mathbf{M}$ depends only on the parameters of the transformations. Linear maps preserve thus the geometrical compatibility of closed strips. This is not a surprise, since linear maps preserve PQ-meshes (Pottmann et al. 2007).
Combescure maps We give now another set of transformations that preserve the geometrical compatiblity. We re-write now equation (21) by using the properties of area of triangles:

$$
a_{i}=\frac{\|\mathbf{B C}\| \sin \gamma}{\|\mathbf{A D}\| \sin \alpha}
$$

Computing the product of all these values, we notice that the lengths cancel out (each length is exactly one time at the numerator and one time at the denominator, so that:

$$
\prod_{i=0}^{N-1} a_{i}=\prod_{i=0}^{N-1} \frac{\sin \gamma_{i}}{\sin \alpha_{i}}
$$

Therefore, a transformation that preserves discrete angles preserves also the geometrical compatibility. Such transformations are known as Combescure transformation. The image of a mesh by a Combescure transformation has its edges parallel to the initial mesh, but it does not necessary preserve lengths. Examples of such transformations are given in Mesnil et al. (2015).

\section{A.5 Particular cases}

We give three simple examples where equation (24) is verified.

Example 1: parallel edges Equation (24) is verified when all the $a_{k}$ are equal to one. This condition translates into:

$$
\frac{\|\mathbf{A C}\|}{\|\mathbf{A D}\|}=\frac{\sin \beta}{\sin \alpha}
$$

We write the equation of $\mathbf{A}, \mathbf{B}, \mathbf{C}, \mathbf{D}$ in the cartesian plane where $\mathbf{e}_{\mathbf{X}}$ is parallel to $\mathbf{A B}$, we have:

$$
\left\{\begin{array}{c}
\mathbf{A B}=\left(\begin{array}{c}
\|\mathbf{A B}\| \\
0 \\
0
\end{array}\right) \\
\mathbf{A C}=\|\mathbf{A C}\|\left(\begin{array}{c}
\cos \beta \\
\sin \beta \\
0
\end{array}\right) \\
\mathbf{A D}=\|\mathbf{A D}\|\left(\begin{array}{c}
\cos \alpha \\
\sin \alpha \\
0
\end{array}\right)
\end{array}\right.
$$

We plug then equation (29) into equation (30) and compute the vector CD. We get following result:

$$
\mathbf{C D}=\|\mathbf{A D}\|\left(\begin{array}{c}
\frac{\sin \alpha}{\sin \beta} \cos \beta-\cos \alpha \\
0 \\
0
\end{array}\right)
$$


Remarkably, we notice that the vectors $\mathbf{C D}$ and $\mathrm{AB}$ are parallel. Reciprocally, if these two vectors are parallel, then equation (29) is satisfied. Therefore, a closed strip where all the projected quads are trapezoids satisfies equation (24). Such planar projections provide thus a large design space and the maximal design flexibility for closed strips.

Example 2: symmetry Consider the case where the planar projection of the strip has an axis of symmetry. Consider Figure 19: each $a_{i}$ is defined as the ratio of the area of the blue and orange triangles. When the curve has a symmetry, like the one depicted in Figure 20, the role of orange and blue triangle is inverted by the symmetry. Two faces related by a symmetry have therefore inverse values of $a_{i}$. Their product is naturally equal to 1 , which proves that strips with an axis of symmetry satisfy equation (24).

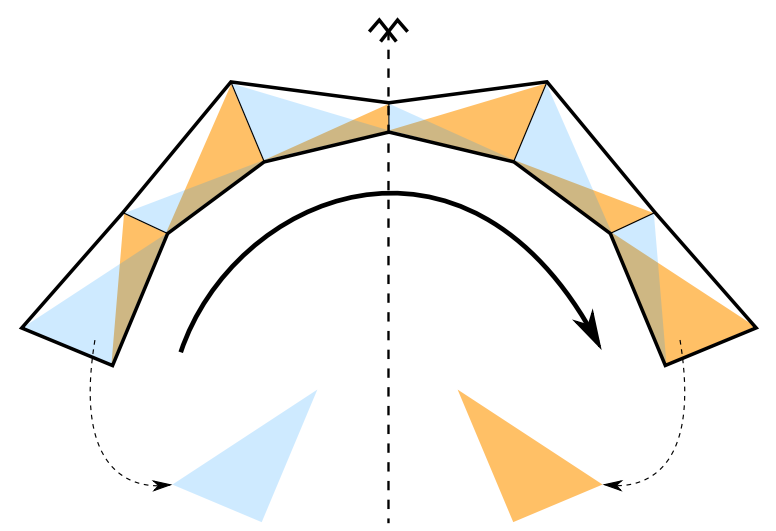

Figure 20. A curve with an axis of symmetry and the inversion of the blue and orange triangles.

Example 3: orthogonal fields The first two examples are based on equation (24) where the $a_{i}$ are expressed as ratios of areas. The propagation rule is applied to each quadrilateral, but in the case of closed curves it is also interesting to look at each vertex.

Consider Figure 21, equation (28) is verified if $\gamma_{0}=\alpha_{1}$, $\gamma_{1}=\alpha_{2}$ and so forth. In other terms, if the transverse edge is the bisecting line of the inner curve, then we have a solution to the problem of closed strips. This condition is a discrete counterpart of orthogonality of vector fields. Examples of such meshes are obtained by moulding surfaces or Monge's surfaces (Mesnil et al. 2015). Discretisation of orthogonal parameterisation of the plane will therefore yield strips that are very close to be geometrically compatible. The smooth counterpart of this problem would be looked at carefully in further work.

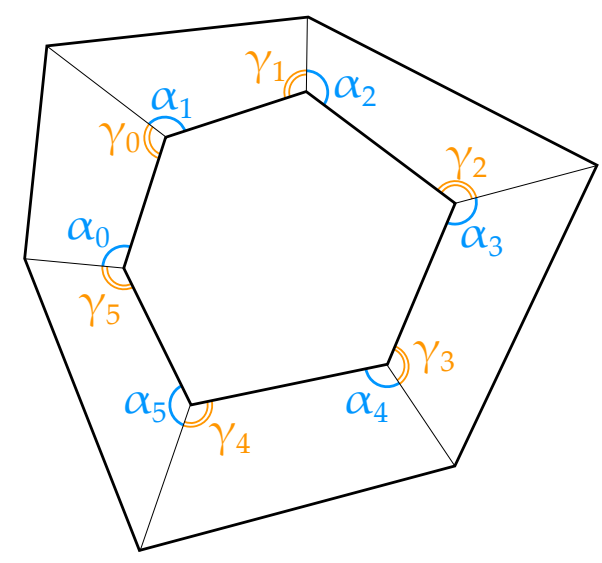

Figure 21. A closed curve and the angles used in equation (28)

\section{B Size of the design space for periodic patterns}

We propose here to count the number of degrees of freedom for the meshes drawn in Figure 16. We use equation (16) to estimate the available degrees of freedom and compare this number with the number of prescribed points drawn in Figure 16. Each time, it is easy to propagate the heights in the manner of what has been done with quadrilateral meshes. We illustrate here the fact that the formula (16) is exact for meshes with no closed curves.

Kagome pattern The Kagome pattern shown in Figure 16a features 191 vertices, 112 triangles, 48 hexagons and 8 pentagons. The estimated number of degrees of freedom given by the marionette method follows:

$$
\begin{gathered}
N=191-(112 \cdot(3-3)+48 \cdot(6-3)+8 \cdot(5-3)) \\
N=191-(0+144+16) \\
N=31
\end{gathered}
$$

This is exactly the number of prescribed nodes in Figure 16a.

Dual-Kagome pattern The dual-Kagome pattern shown in Figure 16b has 185 vertices and 156 quadrilateral facets.

$$
N=185-156 \cdot(4-3)
$$

$$
N=29
$$

This is the number of vertices with prescribed heights in Figure 16b.

Hexagonal pattern The pattern derived from an hexagonal mesh has 183 vertices and 162 quadrilateral facets. The 
number of d.o.f is thus:

$$
N=183-162=21
$$

Remark The calculation provided here shows hints for the number of guide curves to use to lift the mesh. Notice however that the d.o.f must be uncoupled: for example it is not possible to prescribe independently the four heights of the vertices of a quadrilateral face. Our choice of guide points does not violate this constraint.

For both the dual Kagome and the hexagonal pattern, the planarity constraint cannot be propagated throughout the whole mesh, additional isolated heights have to be prescribed. 